\title{
Imagem corporal de adolescentes: um estudo sobre as relações de gênero e influência da mídia
}

\author{
Body image of adolescents: as study on gender relations and influence of the \\ mass media
}

\section{Imagen corporal de adolescentes: un estudio sobre las relaciones de género e influencia de los medios de comunicación de masa}

\author{
Vivianne Oliveira Gonçalves ${ }^{l}$ \\ Juan Parra Martínez $z^{2}$ \\ http://dx.doi.org/10.5216/31792
}

\section{Resumo}

O objetivo do estudo é conhecer as diferenças de gênero em uma amostra de adolescentes em relação à percepção da imagem corporal e percepção da influência da mídia, através de uma abordagem quantiqualitativa. Foram utilizados o Body Shape Questionnaire (BSQ), o Cuestionario de Influencias del Modelo Estético Corporal (CIMEC-26) e o grupo de discussão como técnica de coleta de dados. Os resultados indicam a existência de uma maior insatisfação corporal, assim como maior influência da mídia em adolescentes do sexo feminino. Além disso, os discursos dos grupos de discussão ressaltam uma grande variedade de mediadores na construção da imagem corporal na atualidade: a norma da magreza, a inexistência de uma diversidade de roupas que se adaptem aos diferentes corpos, o impacto da influencia da mídia e a evidência social de que as pessoas magras são mais aceitas em todas as esferas sociais.

Palavras-chave: Imagem corporal. Adolescência. Gênero. Meios de comunicação de massa.

\begin{abstract}
The purpose of the study is to analyze gender differences in a sample of adolescents regarding the perception of body image, perception of the influence of the media and the practice of physical activity. We used the Body Shape Questionnaire (BSQ), the Cuestionario de Influencias del Modelo Estético Corporal (CIMEC-26) and the discussion group as data collection technique. The results indicate the existence of a greater body dissatisfaction, as well as greater influence of mass media on females adolescents. In addition, the speeches of focus groups underscore a wide variety of mediators in the construction of body image today: the norm of thinness, the lack of a diversity of clothes that adapt to different bodies, the impact of the influence of media and social evidence that thin people are more accepted in all social spheres.
\end{abstract}

Keywords: Body image. Gender. Adolescent. Mass media.

\footnotetext{
${ }^{1}$ Doutor em Ciencias del Deporte. Universidad de Castilla-La Mancha, UCLM, Espanha. Docente dos cursos de Educação Física da Universidade Federal de Goiás. Brasil, Goiás, Jataí. E-mail: nanifef@ hotmail.com

${ }^{2}$ Doutor em Ciências da Educação pela Faculdade de Educação, da Universidade Nacional de Educação à Distância (UNED-Madrid). Professor Associado dos Programas de Pós-Graduação da Faculdade de Ciências da Atividade Física e do Esporte, da Universidade de Castilla-La Mancha. Espanha, Toledo. E-mail: juan.parra@uclm.es
}

Comun. \& Inf., Goiânia, GO, v. 17, n. 2, p. 139-154, jul./dez. 2014 


\section{Resumen}

El objetivo del estudio es conocer las diferencias de género en una muestra de adolescentes respecto a la percepción de la imagen corporal y la percepción de la influencia de los medios de comunicación, desde una perspectiva cuantitativa y cualitativa. Fueron utilizados el Body Shape Questionnaire (BSQ), el Cuestionario de Influencias del Modelo Estético Corporal (CIMEC-26) y el grupo de discusión como técnica de recogida de los datos. Los resultados indican la existencia de una mayor insatisfacción corporal en adolescentes del sexo femenino. Además, los discursos de los grupos de discusión resaltan una gran variedad de mediadores en la construcción de la imagen corporal en la actualidad: la norma de la delgadez, la inexistencia de la diversidad de tallas que se adapten a diferentes cuerpos, el impacto de la influencia de los medios de comunicación, y la evidencia social de que las personas delgadas son mejor aceptadas en todos los ámbitos sociales.

Palabras clave: Imagen corporal. Adolescentes. Género. Medios de comunicación de masa.

\section{INTRODUÇÃO}

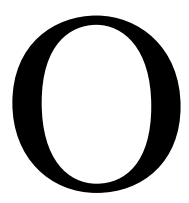

estudo da imagem corporal é relativamente recente, apesar de que a preocupação com a aparência física exista desde o início das civilizações. Podem-se encontrar investigações a respeito na área da neurologia, psicologia, educação física,

reabilitação, entre outras. Desde a década passada vem aumentando o número de pesquisas na área, principalmente devido ao aumento da obesidade e da incidência de transtornos alimentares. Nos últimos anos também se encontram investigações realizadas com pessoas sem transtornos, o que evidencia que a cultura atual vem promovendo um ideal corporal de magreza e, em consequência, uma quantidade importante de pessoas apresenta insatisfação com a forma elou o peso corporal.

A existência desse ideal de beleza, criado e socialmente compartilhado, é uma pressão significativa para a população em geral e especialmente para o adolescente que está em fase de integração da imagem corporal. A adolescência é caracterizada por grandes transformações biológicas, emocionais e sociais verificadas na segunda década da vida, quando o indivíduo passa a adotar comportamentos e práticas diferenciados, caracterizados principalmente pela autonomia e maior exposição às situações do cotidiano. Representa a fase de maior velocidade de crescimento na vida extrauterina, implicando alterações no tamanho, na aparência e na satisfação corporal (AYENSA; GUILLÉN; GARRIDO, 2002). Dessa forma, a aceitação do próprio corpo na adolescência está condicionada por critérios que prevalecem no grupo de amigos, que são, por sua vez, determinados pelos modelos sociais vigentes. Os adolescentes são pressionados por múltiplas instâncias da sociedade: publicidade, revistas, televisão, família e amigos, que lhes propõe um corpo magro e esbelto, especialmente no âmbito feminino. Assim, a insatisfação com a aparência física pode ter como consequência 
uma imagem negativa e distorcida do próprio corpo que, na sua vertente mais problemática, tornar-se um dos sintomas de transtornos alimentares (PÉREZ MANUEL, 2004).

Em relação à imagem corporal, Schilder (1999) a define como a representação mental do corpo que cada um constrói em sua mente. Atualmente, é entendida como um constructo multidimensional que inclui a dimensão perceptual (precisão na percepção do corpo), a dimensão cognitivo-afetiva ou subjetiva (atitudes, sentimentos, cognições e avaliações em relação ao tamanho, peso ou partes do corpo) e a dimensão comportamental (comportamentos provocados a partir da percepção e dos sentimentos com relação ao próprio corpo) (RAICH, 2000).

Diferentes pesquisas têm analisado diversos indicadores que influenciam a imagem corporal. De um lado, os fatores biológicos, tais como a idade (SCHERER et al., 2010; PETROSKI, PELEGRINI e GLANER, 2012), o sexo (BULLY, ELOSUA e LÓPEZJÁUREGUI, 2012), a etnia (REDDY e CROWTHER, 2007) e o índice de massa corporal, (MOTA et al, 2012), entre outros. Por outro lado, os fatores culturais e psicológicos como o autoconceito e a autoestima (DAMASCENO et al., 2011), a família e os amigos (LÓPEZ, CORONA e HALFOND, 2013), os meios de comunicação (CARLSON JONES, 2004) e a prática de atividade física (FORTES et al., 2011).

Essas pesquisas têm utilizado várias medidas para avaliação da imagem de corpo. Os instrumentos mais usados são os questionários e as escalas de silhueta, devido a sua praticidade de aplicação e correção. No entanto, apesar do grande número de instrumentos de avaliação da imagem corporal, o investigador deve especificar bem qual dimensão pretende avaliar e utilizar vários instrumentos ou a combinação de métodos quantitativos e qualitativos (FABRA e DOMÈNECH, 2001).

O objetivo do estudo é conhecer as diferenças de gênero em uma amostra de adolescentes em relação à percepção da imagem corporal e percepção da influência da mídia, através de uma abordagem quanti-qualitativa. $\mathrm{O}$ interesse por esta investigação se relaciona com a importância do ideal estético corporal propagado pela mídia e sua influência na imagem corporal dos adolescentes. Considera-se pertinente complementar os estudos já realizados sobre o tema, enfocando especificamente a questão do gênero a partir de uma abordagem mista de pesquisa.

\section{MÉTODO}


A pesquisa caracteriza-se como quanti-qualitativa, do tipo descritiva. A escolha da amostra foi realizada mediante amostragem por conveniência. A fase quantitativa da pesquisa constitui-se por um total de 237 adolescentes (50,2\% de mulheres e 49,8\% de homens) de distintos centros educativos de Toledo (Espanha), sendo que 27,4\% estavam cursando $3^{\circ}$ de Educación Secundaria Obligatoria (ESO), 29,1\% cursavam $4^{\circ}$ de ESO e 43,5\% cursavam $1^{\circ}$ Bachillerato (BACH). A idade dos adolescentes compreendeu o intervalo de 14 a 18 anos.

Dentro da amostra anterior, e para a fase qualitativa da pesquisa, formou-se um segundo grupo amostral constituído por 60 alunos, sendo que $40 \%$ cursavam $3^{\circ}$ de ESO, $20 \%$ cursavam $4^{\circ}$ de ESO e $40 \%$ estavam cursando Bachillerato.

\subsection{Instrumentos de coleta e análise dos dados}

$\mathrm{Na}$ fase quantitativa da pesquisa, foram aplicados os seguintes instrumentos em suas versões adaptadas e validadas ao espanhol:

1) Body Shape Questionnaire (BSQ-34): desenvolvido por Cooper et al. (1997) e validado por Raich, Torras y Figueras (1996). É composto de 34 itens, sendo que cada item apresenta seis possibilidades de resposta (escala Likert), com um intervalo de 34-204. A partir da pontuação total podem-se classificar os sujeitos da pesquisa nas seguintes categorias de insatisfação corporal: não insatisfação (< 81 pontos), leve insatisfação (81-110 pontos), moderada insatisfação (111-140 pontos), extrema insatisfação (>140 pontos). Segundo a adaptação espanhola, os itens do questionário podem se dividir em cinco fatores: preocupação com o peso em relação à ingestão (PPCI); preocupação pelos aspectos antiestéticos da obesidade (PAAO); insatisfação e preocupação corporal geral (IPCG); insatisfação corporal com a parte inferior do corpo (coxas, quadril e nádegas) (ICPI); e emprego do vômito ou uso de laxantes para reduzir a insatisfação corporal (EVL).

2) Cuestionario de Influencias del Modelo Estético Corporal (CIMEC-26): desenvolvido por Toro, Salamero e Martínez (1994). Composto por 40 perguntas, com uma escala de três pontos, que avaliam a ansiedade pela imagem corporal e o impacto dos modelos sociais e das situações sociais. Uma pontuação maior indica maior influência dos modelos sociais. Possui cinco fatores: preocupação ou mal-estar com a imagem corporal (MIC) (ansiedade ao ter que enfrentar situações que levam ao questionamento do próprio corpo ou que propiciam o desejo de igualar-se aos modelos de referência); influência da publicidade (IP) (interesse que desperta a publicidade de produtos para emagrecer); influência das mensagens verbais (IMV)

Comun. \& Inf., Goiânia, GO, v. 17, n. 2, p. 136-156, jul./dez. 2014 
(interesse que suscitam artigos, livros e conversas sobre emagrecimento); influência dos modelos sociais (IMS) (interesse que despertam os corpos dos modelos publicitários ou de referência); e influência das situações sociais (ISS) (pressão social subjetiva experimentada em determinadas situações e aceitação social da magreza).

$\mathrm{Na}$ fase qualitativa do estudo, foram constituídos 10 grupos de discussão, um por gênero e por ciclo educativo. O número de participantes em cada grupo foi de seis. Foi elaborado um roteiro com perguntas abertas, com uma lista de perguntas relacionadas à imagem corporal e influência da mídia.

Os dados obtidos na fase quantitativa foram analisados com o programa SPSS, versão 17.0 para Windows. Realizaram-se as análises estatísticas e os resultados foram organizados sob a perspectiva de gênero. Os dados da fase qualitativa foram analisados com o programa Atlas ti.

\subsection{Resultados e discussão}

Optamos por apresentar e discutir os dados coletados, buscando analisá-los de forma quantitativa e qualitativa, relacionando-os com o referencial teórico. Assim, apresentamos os resultados tendo como eixo as categorias que emergiram da análise de conteúdos dos discursos produzidos nos grupos de discussão: imagem corporal e percepção da influencia da mídia. Além disso, com relação aos dados qualitativos, iniciamos a apresentação dos resultados de cada subcategoria com uma tabela que mostra os códigos que a compõe e o número de vezes que aparecem no discurso, o que nos permite ter uma ideia da relevância de cada uma das subcategorias para os sujeitos da pesquisa.

\subsection{Imagem corporal}

$\mathrm{Na}$ fase quantitativa, a imagem corporal foi avaliada através do BSQ-34. Os resultados demostram que a maioria dos participantes não apresenta insatisfação com a imagem corporal, no entanto, pode-se observar que as mulheres pontuam mais nas categorias de insatisfação em seus diferentes níveis (Tabela 1).

Tabela 1 - Porcentagem nas categorias do BSQ-34

\begin{tabular}{ccccccc}
\hline Categorias de insatisfação & \multicolumn{3}{c}{ masculino } & \multicolumn{3}{c}{ feminino } \\
\cline { 2 - 6 } Corporal & $\%$ & $\boldsymbol{X}^{\mathbf{2}}$ & $\boldsymbol{p}$ & $\boldsymbol{\%}$ & $\boldsymbol{X}^{\mathbf{2}}$ & $\boldsymbol{p}$ \\
\hline Não apresenta insatisfação & 85,7 & & 0,000 & 55,1 & & 0,000 \\
\cline { 3 - 4 } & & & & & &
\end{tabular}




\begin{tabular}{lcccccc}
\hline \multicolumn{1}{c}{ Categorias de insatisfação } & \multicolumn{3}{c}{ masculino } & \multicolumn{3}{c}{ feminino } \\
\cline { 2 - 6 } \multicolumn{1}{c}{ Corporal } & $\boldsymbol{\%}$ & $\boldsymbol{X}^{\mathbf{2}}$ & $\boldsymbol{p}$ & $\boldsymbol{\%}$ & $\boldsymbol{X}^{\mathbf{2}}$ & $\boldsymbol{p}$ \\
\hline Leve insatisfação & 7,6 & & & 24,6 & 44,92 \\
Moderada insatisfação & 4,2 & 27,50 & & 8,5 & \\
Extrema insatisfação & 2,5 & & & 11,9 & \\
& & & & & \\
\hline
\end{tabular}

Fonte: Elaboração própria.

Da mesma forma, ao comparar as médias obtidas em relação aos fatores do BSQ-34 de acordo ao gênero, encontramos que as adolescentes do sexo feminino alcançam uma pontuação maior que os adolescentes do sexo masculino (Tabela 2).

Tabela 2 - Médias dos fatores do BSQ-34 de acordo com o sexo - U de Mann-Whitney

\begin{tabular}{ccccccc}
\hline Fatores do & \multicolumn{2}{c}{ masculino } & \multicolumn{2}{c}{ feminino } & \\
\cline { 2 - 7 } BSQ & Média & DP & Média & DP & $\boldsymbol{U}$ & $\boldsymbol{p}$ \\
\hline PPCI & 1,66 & 0,93 & 2,51 & 0,11 & 3703,00 & 0,000 \\
PAAO & 1,77 & 0,92 & 2,82 & 0,12 & 3350,50 & 0,000 \\
IPCG & 1,56 & 0,84 & 2,19 & 0,10 & 4235,00 & 0,000 \\
ICPI & 1,71 & 1,02 & 2,95 & 0,14 & 3493,00 & 0,000 \\
EVL & 1,58 & 1,07 & 2,16 & 0,12 & 5027,00 & 0,000 \\
\hline
\end{tabular}

Fonte: Elaboração própria ${ }^{3}$.

Resultados semelhantes foram encontrados no estudo de Ayensa, Guillén e Garrido (2002) realizado com alunos e alunas adolescentes (com idades entre 15 e 19 anos) de ESO de Navarra, na Espanha, utilizando o BSQ-34 como instrumento de coleta de dados. Os resultados mostram diferenças significativas de acordo com o gênero, indicando que as mulheres apresentam maior insatisfação corporal.

No Brasil, a pesquisa desenvolvida por Laus, Costa e Almeida (2009) com 118 estudantes, com idade média de 16,5 anos, com o objetivo de comparar dois instrumentos classicamente utilizados pela literatura especializada (o BSQ-34 e a Escala de Silhuetas) indicados para avaliar possíveis distorções da imagem corporal em adolescentes e adultos de ambos os sexos, encontrou que $14,5 \%$ dos meninos e $60,7 \%$ das meninas apresentaram algum grau de distorção da imagem corporal.

\footnotetext{
${ }^{3} \mathrm{PPCI}=$ preocupação com o peso em relação à ingestão, $\mathrm{PAAO}=$ preocupação pelos aspectos antiestéticos da obesidade, IPCG=insatisfação e preocupação corporal geral, ICPI=insatisfação corporal com a parte inferior do corpo (coxas, quadril e nádegas), e EVL=emprego do vômito ou uso de laxantes para reduzir a insatisfação corporal.
}

Comun. \& Inf., Goiânia, GO, v. 17, n. 2, p. 136-156, jul./dez. 2014 
Segundo Raichet al. (1996), já se têm considerado que a presença da insatisfação corporal, especialmente entre as mulheres, é um fato comum na atualidade, tendo em vista que a cultura atual demanda às mulheres uma crescente preocupação com relação à representação da beleza feminina em ideais que são limitados à aparência física. A cultura popular e a mídia também ajudam a perpetuar esta condição de insatisfação com o próprio corpo. Assim, as adolescentes percebem que a beleza feminina é altamente valorizada pela sociedade e que as mulheres mais bonitas parecem ter mais oportunidades.

A insatisfação corporal encontrada no sexo feminino é corroborada pelos resultados do estudo qualitativo. A categoria imagem corporal na fase qualitativa da pesquisa é composta pelas subcategorias percepção do próprio corpo, percepção do corpo do outro, preocupação com a forma física, e cuidados com o corpo. A Tabela 3 apresenta os códigos que compõe a subcategoria percepção do próprio corpo e a frequência de aparição no discurso.

Tabela 3 - Códigos da subcategoria percepção do próprio corpo

\begin{tabular}{ccc}
\hline Código & Descrição & Frequência \\
SIC & Sentimentos em relação ao próprio corpo & 18 \\
CIC & Mudanças que gostaria de fazer no corpo & 06 \\
IAC & Insatisfação com áreas específicas do corpo & 28
\end{tabular}

Fonte: Elaboração própria

Ressaltamos que são as adolescentes mulheres que relataram maior insatisfação corporal de forma geral e com o peso, com a parte inferior e com a parte mediana do corpo. Já os adolescentes do sexo masculino manifestaram insatisfação com o peso e o tônus muscular. No entanto, observa-se que a insatisfação corporal nos homens relaciona-se com o desejo de ganhar peso e musculatura, enquanto que nas mulheres o desejo é de perder peso. De forma os adolescentes do sexo masculino dizem sentir-se bem, o que atribuem à prática de atividade física e a um estilo de vida saudável.

Os adolescentes também se mostraram conscientes das diferenças relacionadas à percepção da imagem corporal de acordo com o gênero.

Lo que pasa es que los chicos cambian menos que las chicas. $\left(1^{\circ} \mathrm{BACH}\right.$ feminino)

Es que si te pones a pensar todo el tiempo te están diciendo lo que tienes que hacer y eso... en casa, en el Instituto, en las revistas, en todas partes. $\left(3^{\circ}\right.$ ESO feminino)

Yo no creo que sea así, pero es verdad que hay eso de cuídate, y una chica no debe hacer esto y lo otro y no bebas y no fumes y ten cuidado. ( $3^{\circ} \mathrm{ESO}$ feminino). 
A esse respeito Toro e Vilardell (1987) ressaltam que as exigências atuais são distintas de acordo com o gênero. Por exemplo, a cultura atual exige que as mulheres comam de maneira diferente. Comer muito ou inclusive em quantidade moderada em público pode ser considerado inapropriado. Já com o homem sucede o contrário: a quantidade de comida ingerida está associada com sua masculinidade, seu poder e sua força. A forma corporal exigida também é diferente em ambos os casos. Apesar de que a sociedade siga sem aceitar a obesidade, o volume corporal e muscular conota o valor positivo da potência física do homem.

$\mathrm{Na}$ subcategoria percepção do corpo do outro (Tabela 4), os adolescentes se expressam a respeito do corpo do outro, o que nos ajuda a desvelar outros aspectos relativos às relações de gênero. Schilder (1999) já afirmava que há uma conexão estreita entre nossa imagem de corpo e a imagem corporal das outras pessoas. A esse respeito, Williams e Bendelow (1998) apontam que a principal contribuição de Schilderé a ênfase nos aspectos sociais, já que a imagem de corpo é desenvolvida e construída nas relações sociais e através destas.

Tabela 4 - Códigos da subcategoria percepção do corpo do outro

\begin{tabular}{ccc}
\hline Código & Descrição & Frequência \\
\hline PGF & Percepção do gênero feminino & 12 \\
PGM & Percepção do gênero masculino & 10 \\
IGF & Interesse pelo gênero feminino & 15 \\
IGM & Interesse pelo gênero masculino & 19 \\
\hline
\end{tabular}

Fonte: Elaboração própria

A partir da própria experiência pessoal e de relação com seus amigos do mesmo sexo ou do sexo oposto, os adolescentes apontam os valores que articulam e organizam as atitudes e interesses de ambos. Dessa forma, apresentam os estereótipos que os mobilizam, assim como os atributos assignados a cada gênero.

A ver, es que a las mujeres les exigen que sean guapas y delgadas, mucho más que los chicos. ( $3^{\circ} \mathrm{ESO}$ masculino)

Pues deportistas, lo que decían ellas, somos mucho más deportistas que ellas. $\left(1^{\circ} \mathrm{BACH}\right.$ masculino)

Yo no creo que sea así, pero es verdad que hay eso de cuídate, y una chica no debe hacer esto y lo otro y no bebas y no fumes y ten cuidado. $\left(3^{\circ} \mathrm{ESO}\right.$ feminino)

A maioria dos adolescentes que participaram desse estudo reconheceu que essas diferenças entre os gêneros foram atribuídas social e culturalmente. De fato, a sociedade 
submete a homens e a mulheres a determinados estereótipos de gênero, descrevendo e prescrevendo suas qualidades e suas condutas, ou seja, como devem ser e o que devem fazer.

A Tabela 5 mostra os códigos que compõe a subcategoria preocupação com a forma física.

Tabela 5 - Códigos da subcategoria preocupação com a forma física

\begin{tabular}{ccc}
\hline Código & Descrição & Frequência \\
\hline PFF & Preocupação com a forma física & 10 \\
EPF & Excessiva preocupação forma física & 29 \\
PFG & Preocupação com a forma física de acordo com o gênero & 08 \\
\hline
\end{tabular}

Fonte: Elaboração própria

A opinião dos participantes, de ambos os sexos é a de que as mulheres apresentam maior preocupação.

Suelen preocuparse con lo que va a ver el otro, por lo que dirán. ( $1^{\circ}$ Bach masculino)

Porque desde pequeñitas las están haciendo ver como que tienen que ser más guapas, están más preocupadas con eso, mientras que los hombres se conforman. ( $4^{\circ}$ ESO masculino)

Isso acontece, segundo Etcoff, Orbach e Scout (2004), porque as mulheres veem a beleza e os atributos físicos como altamente demandados e reconhecidos, sentem a pressão social para serem fisicamente atraentes, ou seja, sofrem maiores diretrizes dos diferentes âmbitos sociais para melhorar cada vez mais a sua aparência.

A questão dos excessos também é abordada pelos adolescentes. A maioria afirma conhecer alguém que apresenta excessiva preocupação com a imagem do corpo, o que as leva a fazer dietas, deixar de comer ou fazer exercícios físicos de forma compulsiva.

No discurso dos participantes dos grupos de discussão, referente aos cuidados com o corpo (Tabela 6), destaca-se a prática de atividade física, a dieta e o uso de cosméticos e maquiagem, ou a combinação das anteriores. No que se refere às diferenças de gênero, as mulheres fazem mais dieta e os homens dedicam-se mais ao exercício físico.

Tabela 6 - Códigos da subcategoria cuidados com o corpo

\begin{tabular}{ccc}
\hline Código & Descrição & Frequência \\
\hline CRE & Cosméticos e maquiagem & 04 \\
CUD & Dieta & 16 \\
CUE & Prática de exercício físico & 10 \\
OPP & Operações plásticas & 07 \\
\hline
\end{tabular}

Fonte: Elaboração própria 
Com relação à prática de exercícios físicos, o discurso dos participantes confirma a existência de diferenças que se relacionam com os estereótipos atribuídos a cada gênero. Assim, os homens praticam mais o futebol e outros esportes coletivos, enquanto que as mulheres se involucram mais na dança, na natação e na ginástica.

A mí la gimnasia y eso, nada, pero me gusta el fútbol y el baloncesto. ( $4^{\circ}$ ESO masculino).

Yo hacer deporte no hago porque no me gusta, no me gusta nada que tenga que ver con el cansancio, con sudar, con que el cuerpo se canse. $\left(1^{\circ} \mathrm{BACH}\right.$ feminino).

A mí algunos deportes sí que me gustan pero me gustan más verlos que practicarlos. ( $1^{\circ} \mathrm{BACH}$ feminino).

Ainda as respostas sejam pontuais, se confirma a existência de diferenças de gênero e que se relacionam com os estereótipos de gênero. A presença de tais estereótipos pode-se visualizar também se retomamos os estudos já realizados, como os de Cohane e Pope (2001) e Penas-Lledo, Vaz Leal e Waller (2002). Estes autores encontraram que os adolescentes do sexo masculino praticam mais o futebol e os esportes de combate, enquanto que as adolescentes participam de atividades como a dança, a natação, o atletismo e o voleibol. Além disso, apontam que homens e mulheres praticam atividades físico-esportivas por diferentes motivos. Os homens com a finalidade de conseguir maior massa muscular e as mulheres com o objetivo de perder peso.

Tais diferenças também foram encontradas no estudo realizado por Blández Ángel, Fernández García e Zamorano (2007). Os discursos dos adolescentes dos grupos de discussão realizados pelos autores acima citados apontaram que esta diferenciação se vincula a uma orientação estética e recreativa da prática esportiva para as mulheres, enquanto que para os homens a prática se vincula à condição física e visando a performance esportiva.

\subsection{Percepção da Influência da mídia}

Com relação à influência dos modelos sociais avaliada com o CIMEC-26, analisouse inicialmente a pontuação total obtida, levando em consideração o ponto de corte estabelecido em 17 pontos. Os resultados revelam que as adolescentes do sexo feminino são mais influenciadas (33,5\% das adolescentes do sexo feminino e 9,4\% do masculino).

Quando comparamos as diferenças de gênero com relação aos fatores do CIMEC-26, encontramos que as mulheres alcançam pontuações maiores que os homens em todos os fatores (Tabela 7). 
Tabela 7 - Média dos fatores do CIMEC-26 - U de Mann-Whitney

\begin{tabular}{ccccccc}
\hline \multirow{2}{*}{$\begin{array}{c}\text { Fatores do } \\
\text { CIMEC }\end{array}$} & \multicolumn{7}{c}{ masculino } & \multicolumn{2}{c}{ feminino } \\
\cline { 2 - 6 } & Média & DP & Média & DP & $\boldsymbol{U}$ & $\boldsymbol{P}$ \\
\hline MIC & 0,45 & 0,39 & 0,83 & 0,46 & 3564,00 & 0,000 \\
IP & 0,24 & 0,28 & 0,47 & 0,40 & 4381,50 & 0,000 \\
IMV & 0,20 & 0,27 & 0,49 & 0,45 & 4222,00 & 0,000 \\
IMS & 0,46 & 0,45 & 0,66 & 0,46 & 5255,50 & 0,001 \\
ISS & 0,43 & 0,34 & 0,51 & 0,30 & 5983,00 & 0,043
\end{tabular}

Nota: elaboração própria ${ }^{4}$.

Alguns estudos realizados recentemente (CARLSON JONES, 2004; GRABE, WARD e HYDE, 2008) sugerem que existe uma maior inconformidade com o peso no sexo feminino em relação ao masculino, assim como uma maior interiorização do modelo estético de referência, o que leva à comparação com sua própria imagem corporal, coincidente com os resultados encontrados em nossa pesquisa. A esse respeito, a investigação realizada por Fernández, Otero e Rodríguez Castro (2004) alerta que o risco de desenvolver um transtorno alimentar em mulheres não se vincula com o consumo dos meios de comunicação, ou seja, com a frequência de uso destes, mas com o tipo de conteúdo que se vê na televisão e que se lê nas revistas.

Ressalta-se o estudo realizado por Botta (1999), com o objetivo de medir o impacto das imagens dos meios de comunicação na imagem corporal em adolescentes do sexo feminino. Os resultados mostraram que os meios de comunicação têm grande influência no corpo que aspiravam ter essas adolescentes. Estas percebiam os corpos das modelos e as imagens femininas como realistas e uma de suas metas era poder conseguir corpos semelhantes. Assim, os investigadores reafirmam a importância da exposição aos meios de comunicação, sua influência e a percepção social que se criou em torno a um ideal de corpo magro e perfeito como modelo básico de beleza feminina.

Para conhecer a associação entre a influência dos modelos sociais e a insatisfação corporal, realizou-se uma análise de correlação de Sperman. Os resultados mostraram uma correlação positiva, moderada e alta entre o BSQ-34 e o CIMEC-26, o que evidencia que o aumento da insatisfação corporal relaciona-se com o aumento da influência dos modelos estéticos e sociais (Tabela 8).

\footnotetext{
${ }^{4} \mathrm{MIC}=$ mal-estar com a imagem corporal, IP=influência da publicidade, IMS=influência das mensagens verbais, IMS=influência dos modelos sociais, e ISS=influência das situações sociais.
} 
Tabela 8 - Correlação entre o CIMEC-26 e o BSQ-34 de acordo ao sexo

\begin{tabular}{|c|c|c|}
\hline & masculino & CIMEC-26 \\
\hline & Correlação de Pearson & 0,530 \\
\hline \multirow[t]{4}{*}{$B S Q-34$} & Sig. (bilateral) & 0,000 \\
\hline & $\mathrm{N}$ & 189 \\
\hline & feminino & CIMEC-26 \\
\hline & Correlação de Pearson & 0,670 \\
\hline \multirow[t]{2}{*}{$B S Q-34$} & Sig. (bilateral) & 0,000 \\
\hline & $\mathrm{N}$ & 188 \\
\hline
\end{tabular}

Fonte: Elaboração própria

$\mathrm{Na}$ fase qualitativa da pesquisa, a esta categoria foram relacionados três conceitos ou subcategorias: padrão corporal na sociedade de consumo, protótipos corporais e influência da mídia. Os códigos da subcategoria padrão corporal na sociedade de consumo são mostrados na Tabela 9.

Tabela 9 - Códigos da subcategoria padrão corporal na sociedade de consumo

\begin{tabular}{ccc}
\hline Código & Descrição & Frequência \\
\hline NDE & Norma da magreza & 21 \\
TVB & Beleza física e vantagem profissional & 37 \\
TLL & O tamanho das roupas & 13 \\
\hline
\end{tabular}

Fonte: Elaboração própria

Em relação à norma da magreza, afirmam que, apesar de que atualmente exista um culto à magreza, tal exigência parece não afetar a todos os países da mesma maneira.

No es lo mismo, porque tú ves a las sudamericanas, yo creo que las sudamericanas tienen más ancho de cadera y así... en general como la sociedad es así, entonces como que... ya lo asumen, intentan no remediarlo, intentan tener las caderas definidas, bonitas, pero no intentan... no están obsesionadas. ( $1^{\circ} \mathrm{BACH}$ feminino)

Os participantes desse estudo opinam que sua cultura é uma cultura obsessionada pelo corpo e sua exposição tecnológica, visual e comercial. Como consequência, existe uma pressão enorme para ser magro (a) e bonito (a), de tal forma que ser feio, e principalmente estar gordo, são obstáculos para o êxito social e profissional.

Pues yo estoy con ella, si eres guapa es más fácil porque las guapas les gustan a todos. ( $3^{\circ} \mathrm{ESO}$ feminino)

Porque las empresas buscan sacar la mejor producción con los mejores medios, a ver. No te van a poner un anuncio que sale un tío más feo, sacando un producto de belleza. ( $1^{\circ} \mathrm{BACH}$ masculino) 
Yo también creo que influye, es difícil que tengas un trabajo si eres gorda, bueno, en la administración sí, porque no te pueden dejar y yo conozco porque mi madre trabaja en la administración, pero en la empresa, seguro que no te cogen. ( $3^{\circ}$ ESO masculino)

Resultados similares a esta pesquisa foram encontrados em uma investigação realizada através de entrevistas e grupos de discussão com relação aos conhecimentos corporais com adolescentes australianas que desejavam emagrecer (TIGGEMANN e WILLIAMSON, (2000). Os resultados indicaram a influências das modelos e dos meios de comunicação, assim como ser bonita e receber mais atenção como as duas principais razões para desejarem ser magras. Constatou-se também que as adolescentes descreveram claramente e de forma articulada como os meios de comunicação e os conceitos de beleza feminina propagados por tais meios se tornam crenças e normas culturais.

Outra questão muito comentada, sobretudo pelas adolescentes mulheres, se refere ao tamanho das roupas. A esse respeito, as jovens apontam que o tamanho das roupas é cada vez menor, além de relatar a não existência de uma diversidade de roupas que possa adaptar-se à diversidade de corpos e o impacto psicológico negativo ao não encontrar o seu tamanho.

Esto ya ha pasado por la tele que cada vez hace la ropa más pequeña y esto es malo, hasta para las modelos. ( $4^{\circ}$ ESO feminino)

Es que da mucha pena cuando quieres comprarte algo y no te entra. $\left(4^{\circ} \mathrm{ESO}\right.$ feminino)

A mí, no me gusta ni me disgusta mi cuerpo, pero que quieres que te diga, si voy a la tienda y no puedo coger la 36 , pues hombre, no me sienta nada bien. ( $4^{\circ}$ ESO feminino)

A este respeito, Urbón Ladrero (2005) afirma que desde princípios dos anos 90, o tamanho das roupas, principalmente as destinadas às mulheres, tem sofrido importantes mudanças e transformações, igualmente sujeitas aos condicionantes econômicos, políticos, sociais e publicitários. Se anteriormente, o tamanho 44 era comercializado com normalidade nas lojas, agora é difícil encontrar o tamanho 42. Por outro lado, o tamanho 38 era o mínimo e se desejasse menor, teria que buscar em lojas especializadas. No entanto, hoje em qualquer loja de moda juvenil se encontra os 36, 34 e até 32, que muitas vezes não corresponde a medidas reais da maioria das adolescentes.

Ressaltamos um estudo realizado em 2008 pelo Ministerio de Sanidad y Consumo espanhol, cujo objetivo era definir três formas prevalecentes de silhueta feminina, com o propósito de unificar o tamanho das roupas em Espanha, que revelou que $80 \%$ das mulheres tinham um índice de massa corporal considerado adequado segundo os parâmetros relativos à 
saúde. No entanto, $40 \%$ das mulheres afirmaram ter problemas para encontrar roupa, e o principal motivo, em todas as idades pesquisadas, foi que a roupa era pequena. Entretanto, na atualidade, o processo de unificação do tamanho das roupas ainda não é uma realidade (FERNÁNDEZ, OTERO e RODRÍGUEZ CASTRO, 2004).

Além disso, na pesquisa mencionada anteriormente, os dados revelam que $44 \%$ das mulheres planejaram fazer uma dieta depois de comprovar que não usam o tamanho da roupa que acreditavam usar. Estes resultados apoiam a teoria de que o sistema de tamanho das roupas atual propicia a insatisfação corporal e tem impacto direto na tomada de decisão de perder peso (FUNDACIÓN, 2010). Por tanto, caberia ao governo tomar medidas mais efetivas com o intuito de unificar o tamanho das roupas e diminuir o impacto da indústria da moda na saúde das pessoas.

A Tabela 10 apresenta os protótipos corporais transmitidos pelos meios de comunicação.

Tabela 10 - Códigos da subcategoria protótipos corporais transmitidos

\begin{tabular}{ccc}
\hline Código & Descrição & Frequência \\
\hline PCM & Protótipos corporais masculinos & 06 \\
PCF & Protótipos corporais femininos & 10 \\
PCG & Protótipos corporais de acordo com o gênero & 05
\end{tabular}

Fonte: Elaboração própria

Nessa subcategoria, os participantes dos grupos de discussão identificam como referência corporal uma complexão atlética para o homem e a magreza para a mulher.

Sí, en la tele siempre vemos las modelos que son perfectas, siempre te las ponen así, que muy delgadas. Y por ejemplo, las modelos siempre tienden a ser muy altas. Las bajitas tú no ves muy monas. $\left(1^{\circ} \mathrm{BACH}\right.$ feminino)

Bueno, varia un poco, pero los chicos, todos son de gimnasio. $\left(1^{\circ} \mathrm{BACH}\right.$ feminino)

Delgadísimas las ponen siempre. Y altísimas, con unas piernas muy altas. Y si son bajitas, más delgadas aún, para que no se note. $\left(1^{\circ}\right.$ Bach masculino)

No que diz respeito à influência da mídia, a análise proposta busca revelar as reflexões dos participantes em relação ao que percebem do universo mediático com o qual convivem cotidianamente (Tabela 11).

Tabela 11 - Códigos da subcategoria influência da mídia

\begin{tabular}{|c|c|c|}
\hline Código & Descrição & Frequência \\
\hline IMM & Percepção da influencia da mída & 32 \\
\hline
\end{tabular}

Comun. \& Inf., Goiânia, GO, v. 17, n. 2, p. 136-156, jul./dez. 2014 


\begin{tabular}{|c|c|c|}
\hline Código & Descrição & Frequência \\
\hline MET & Mensagens transmitidas pela mídia & 20 \\
\hline MAP & Mulheres como principal alvo das mensagens & 07 \\
\hline CIM & Críticas a influencia da mídia na imagem corporal & 12 \\
\hline
\end{tabular}

Fonte: Elaboração própria

Os resultados mostram que os participantes reconhecem a influência da mídia, contudo, afirmam ser inevitável a comparação do próprio corpo com o corpo ideal que é divulgado, o que muitas vezes provoca condutas de perda de peso ou outros cuidadoslintervenções com o corpo.

Yo pienso que los medios de comunicación son muy influenciables, tal como te lo pongan es como te lo vas a creer, entonces si te lo ponen muy bien y te ponen este prototipo de persona, pues es lo que va a gustar a la gente. Y las revistas igual y todo. $\left(1^{\circ}\right.$ Bach femenino)

Claro, porque ves en una revista los cuerpos de las modelos, porque las modelos hay algunas que están excesivamente delgadas, los cuerpos de algunas famosas y eso, y tú la ves y la ves bien, y si como que dices, no "que envidia", sino que podría estar así o me gustaría estar así. ( $1^{\circ} \mathrm{BACH}$ feminino)

Enfatizam ainda que as mulheres são o principal objetivo das mensagens transmitidas. No entanto, ressalta-se que foram os adolescentes do sexo masculino que chamaram a atenção para a visão estereotipada da mulher que aparece na mídia.

Pues por la cultura que tenemos, porque nos dicen los cánones que tienes que seguir, ponen los escaparates, los maniquíes tienen una 38 o una 36 , con un pecho una 90 y tal. Hombre, pues es muy difícil no fijar en esto, porque se está rodeado completamente. Los chicos, no lo sé si es porque tiene menos maniquíes o menos publicidad, así es algo puntual, puede ser que se fijan menos. ( $1^{\circ} \mathrm{BACH}$ feminino)

La televisión lo único que da a las tía es ser materialista, estar delgadísima y perfecta, para que un tío que tenga millones las quiera y no tengan que trabajar. ( $1^{\circ}$ Bach masculino)

Lo que están haciendo son mujeres objeto. Y que no importa lo que sea, si eres guapa $\left(1^{\circ}\right.$ Bach masculino $)$

Autores como Tiggemann e Slater (2004) e Botta (1999) sugerem que a mídia exerce uma influência negativa sobre as mulheres, gerando comparação social com os modelos de beleza apresentados e insatisfação social. Nesse sentido, apontam principalmente a televisão e as revistas como principais promotores.

No entanto, é possível encontrar algum exemplo que representa todo o contrário à tendência geral, como as campanhas de Dove, sustentadas na representação de mulheres reais. 
A campanha iniciou-se como consequência de um estudo realizado em 2004, com 3200 mulheres, de 18 a 64 anos, residentes em cerca de 10 países. Os resultados mostraram que $68 \%$ das mulheres pesquisadas (80\% no caso das espanholas) acreditam que os meios de comunicação e a publicidade divulgam um ideal standard de beleza que a maioria não pode alcançar. Além disso, 75\% (89\% das espanholas) gostariam que tais meios de comunicação mostrassem mulheres com variados tipos físicos (idade, forma do corpo e peso) (FUNDACIÓN, 2010). Estes e outros dados demonstram a necessidade de fugir das representações irreais e promover novos discursos acerca da beleza, que podem impulsionar novas representações e realidades menos estereotipadas.

\section{CONSIDERAÇÕES FINAIS}

A análise da percepção da imagem corporal dos adolescentes participantes demonstrou que o gênero é fundamental no estudo da imagem corporal. Os resultados dessa pesquisa, tanto na fase quantitativa como na fase qualitativa, confirmam os obtidos em outros estudos realizados sobre o tema: indicam a existência de uma maior preocupação e insatisfação com a imagem corporal em adolescentes do sexo feminino.

Os resultados também confirmam a associação entre a insatisfação corporal e a influência da mídia, ao transmitir estereótipos corporais de beleza que afetam principalmente às mulheres. Verificamos que a magreza do corpo aparece conectada a uma diversidade de significados que influem na construção da imagem corporal na atualidade: a associação entre magreza e beleza, e entre beleza e sucesso pessoal e profissional, além da evidência social de que as pessoas magras são aceitas em todos os âmbitos sociais. Como consequência, geram condutas de comparação e insatisfação corporal, apesar de que os modelos de corpo propagados pela mídia sejam representativos de uma realidade distinta a sua própria realidade e composição corporal.

Entretanto, cabe ressaltar que assumir o corpo como uma experiência implica ter uma imagem de si mesmo, a qual se vive interiormente, sozinho, mas também no contexto social em que o indivíduo se desenvolve, onde as opiniões dos demais e os padrões estéticos são muito importantes, principalmente na adolescência. Dessa forma, torna-se necessário questionar a forma como vem sendo processada a informação social relativa às características associadas ao gênero, assim como é necessário refletir acerca das mensagens estereotipadas transmitidas na mídia e suas consequências. 


\section{REFERÊNCIAS}

AYENSA, J. I. B.; GUILLÉN, F.; GARRIDO, E. Insatisfacción corporal en adolescentes medida con el Body Shape Questionnaire (BSQ): efecto del anonimato, el sexo y la edad. International Journal of Clinical and HealthPsychology, v. 2, n.3, p. 439-450, 2002.

BLÁNDEZ ÁNGEL, J.; FERNÁNDEZGARCÍA, E.; ZAMORANO, M. A. Estereotipos de género, actividad física y escuela: la perspectiva del alumnado. Revista de currículum y formación del profesorado, Granada, n. 2, p. 1-21, 2007.

BOTTA, R. A. Television images and adolescent girl's body image disturbance. Journal of Communication, v. 49, n. 2, p. 22-41, 1999.

BULLY, P.; ELOSUA, P.; LÓPEZ JÁUREGUI, A. Insatisfacción corporal en la adolescencia: evolución en una década. Anales de Psicología, v. 28, n. 1, p. 196-202. 2002.

CARLSON JONES, D. Body image among adolescent girls and boys: a longitudinal study. Developmental Psychology, v. 40, n.5, p. 823-835, 2004.

COHANE, G. H.; POPE, J. R. H. G. Body image in boys: a review of the literature. International Journal of Eating Disorders, v. 29, p. 373-379, 2001.

COOPER, P. J. et al. The development and validation of the Body Shape Questionnaire. International Journal of Eating Disorders, v. 6, p. 485-494, 1987.

DAMASCENO et al. Associação entre comportamento alimentar, imagem corporal e esquema de gênero do autoconceito de universitárias praticantes de atividade física. Revista Brasileira de Atividade Física, v. 16, n. 2, p. 138-143, 2011.

ETCOFF, Nancy; ORBACH, Susie; SCOTT, Jennifer. La verdad acerca de la belleza: Resultados del Estudio Global de Dove sobre las Mujeres, la Belleza y el Bienestar. 2004. Disponível em: <http://biblioteca2.ucab.edu.ve/anexos/biblioteca/marc/texto/AAQ6790_Estudio Global de Dove.pdf >. Acesso em: 20 nov. 2010.

FABRA, M. L.; DOMÈNECH, M. Hablar y escuchar. Barcelona: Paidós, 2001.

FERNÁNDEZ, M. L.; OTERO, M. C.; RODRÍGUEZ CASTRO, I. Influencia de la imagen corporal y la autoestima en la experiencia sexual de estudiantes universitarias sin trastornos alimentarios. International Journal of Clinical and Health Psychology, v. 2, p. 357-370, 2004.

FORTES, S. L. et al. Insatisfação corporal de adolescentes atletas e não-atletas. Jornal Brasileiro de Psiquiatria, v. 60, n.4, p. 309-314, 2011.

FUNDACIÓN Imagen y Autoestima. Análisis sobre el grado de unificación de tallas de ropa en España y el impacto en la salud de las personas. Barcelona: Fundación Imagen y Autoestima, 2010. Disponível en <www.f-ima.org>. Acesso em 02 ago. 2010.

GRABE, S.; WARD, L. M.; HYDE, J.S. The role of the media in body image concerns among women: a meta-analysis of experimental and correlational studies. Psychological Bulletin, v. 134, n.3, p. 460-76, 2008.

LAUS, M. F.; COSTA, T. M. B.; ALMEIDA, S. S. Distorção da imagem corporal em adolescentes: um estudo de comparação entre dois instrumentos. Medicina (Ribeirão Preto), v. 42, n.3, p.35-365, 2009.

LOPEZ, V.; CORONA, R.; HALFOND, R. Effects of gender, media influences, and traditional gender role orientation on disordered eating and appearance concerns among Latino adolescents. Journal of Adolescence, v. 36, n.4, p. 727-736, 2013.

Comun. \& Inf., Goiânia, GO, v. 17, n. 2, p. 136-156, jul./dez. 2014 
MOTA, D.C.L. et al. Imagem corporal e sua relação com a atividade física e o estado nutricional de adolescentes. Psico, v. 43, n. 2, p. 237-242, 2012.

PENAS-LLEDO, E.; VAZ LEAL, F. J.; WALLER, G. Excessive exercise in anorexia nervosa and bulimia nervosa: relation to eating characteristics and general psychopathology. International Journal of Eating Disorders, v. 31, n. 4, p. 370-375, 2002.

PÉREZ MANUEL, S. Estado nutricional y psicosocial en adolescentes con alteración de la conducta alimentaria. Tese (Doutorado em Ciência da Saúde) - Facultad de Medicina i Ciències de la Salut, Universitat Rovira i Virgili, 2004.

OLIVEIRA, F. B. Implantação e prática da gestão ambiental: discussão e estudo de caso. 1999. 109f. Dissertação (Mestrado em Engenharia de Produção) - Universida de Federal do Rio Grande do Sul, Porto Alegre, 1999.

PETROSKI, E. L.; PELEGRINI, A.; GLANER, M. F. Motivos e prevalência de insatisfação com a imagem corporal em adolescentes. Ciência e Saúde Coletiva, v. 17, n.4, p. 1071-1077, 2012.

RAICH, R. M. Imagen corporal: conocer y valorar el propio cuerpo. Madrid: Pirámide, 2000.

RAICH, R. M.; TORRAS, J.; FIGUERAS, M. Estudio de la imagen corporal y su relación con el deporte en una muestra de estudiantes universitarios. Análisis y Modificación de Conducta, v. 22, n. 85 , p. 603-626, 1996.

RAICH, R. M. et al. Adaptación de un instrumento de evaluación de la insatisfacción corporal. Clínica y Salud, v. 7, p. 51-66, 1996.

REDDY, S. D.; CROWTHER, J. H. Teasing, acculturation, and cultural conflict: psychosocial correlates of body image and eating attitudes among South Asian women. Cultural Diversity and Ethnic Minority Psychology, v. 13, n. 1, p. 45-53, 2007.

SCHERER, F.C. et al. Imagem corporal em adolescentes: associação com a maturação sexual e sintomas de transtornos alimentares. Jornal Brasileiro de Psiquiatria, v. 59, n. 3, p. 198-202, 2010.

SCHILDER, P. A imagem do corpo: as energias construtivas da psique. São Paulo: Martins Fontes, 1999.

TIGGEMANN, M.; WILLIAMSON, S. The effects of exercise on body satisfaction and self-esteem as a function of gender and age. Sex Roles, v. 43, p. 119-127, 2000.

TORO, J.; SALAMERO, M.; MARTÍNEZ, E. Assesment of sociocultural influences on the aesthetic body shape model in anorexia nervosa. Acta Psychiatr Scand, v. 9, p. 141-151, 1994.

TORO, J.; VILARDELL, E. Anorexia nerviosa. Madrid: Mr Ediciones, 1987.

URBÓN LADRERO, E. La imagen corporal, entre la biología y la cultura: antropología de la alimentación, nutrición y salud. Zainak Cuadernos de Antropología-Etnografía, n. 27, p. 269-278, 2005.

WILLIAMS, S. J.; BENDELOW, G. The lived body: sociological themes, embodied issues. London: Routledge, 1998.

Recebido em: 05/09/2014

Aceito em: 08/10/2014

Publicado em: 10/12/2014

Comun. \& Inf., Goiânia, GO, v. 17, n. 2, p. 136-156, jul./dez. 2014 